\title{
Narrativas de mujeres sobre la maternidad en un contexto de intervención sociosanitaria en la infancia temprana del Chile actual
}

\section{Narratives of women about motherhood in a context of socio-health intervention in early childhood in Chile today}

\author{
Claudia Calquín Donoso ${ }^{a}$ \\ (iD) https://orcid.org/0000-0002-4420-5927 \\ E-mail: claudia.calquinळusach.cl \\ Rodrigo Guerra Arrau ${ }^{b}$ \\ (D) https://orcid.org/0000-0002-0573-6194 \\ E-mail: rodrigo.guerraarrauळgmail.com \\ Sebastián Vásquez ${ }^{\mathrm{a}}$ \\ (D) https://orcid.org/0000-0003-4432-048X \\ E-mail: sebastian.vasquezvœusach.cl \\ aUniversidad de Santiago de Chile. Santiago, Chile. \\ ${ }^{b}$ Universidad Diego Portales. Núcleo Milenio Autoridad y \\ Asimetrías de Poder. Santiago, Chile.
}

\section{Resumen}

Se presentan los resultados de un estudio dirigido a conocer las narrativas sobre la maternidad y el entramado de sentidos atribuidos a las prestaciones de un sistema estatal de atención en salud infantil, en un grupo de usuarias en la ciudad de Santiago de Chile. Por medio de una estrategia cualitativa-narrativa, se exponen tres narrativas sobre la maternidad en el contexto de intervención: conversión, emprendedora y ambivalente. Se concluye que el dispositivo de atención es un referente central en la experiencia de la maternidad en tanto ofrece significados sobre una maternidad ideal y guiada por expertos. Apreciamos que los objetos para la crianza que el sistema proporciona juegan un rol importante para la construcción de los significados en torno a la propia maternidad y a las prestaciones estatales. La multiplicidad de posicionamientos que estas narrativas ofrecen muestra las formas en que las mujeres se autoproducen como madres, pero además las identificaciones con los discursos del sistema, las resistencias, confrontaciones y cuestionamientos, especialmente en el ámbito de la promoción de la lactancia materna.

Palabras clave: Maternidades; Servicios de Salud del Niño; Narración; Desarrollo Infantil; Sistema Chile Crece Contigo.

\section{Correspondencia}

Claudia Calquin Donoso

Av. Ecuador, 3.560, Estación Central. Santiago, Chile. CP 8320000. 
The results of a study aimed at knowing the narratives about motherhood and the network of meanings attributed to the benefits of a state system of child health care, in a group of users in the city of Santiago, Chile, are presented. Through a qualitative-narrative strategy, three narratives about motherhood are presented in the context of intervention: conversion, entrepreneurial and ambivalent. It is concluded that the care device is a central reference in the experience of motherhood as it offers meanings about ideal motherhood and guided by experts. We appreciate that the objects for the upbringing that the system provides, play an important role for the construction of the meanings around the maternity itself and the state benefits. The multiplicity of positions that these narratives offer show the ways in which women self-produce as mothers but also the identifications, resistance, confrontations and questions in key areas for the system itself, such as the promotion of breastfeeding.

Keywords: Maternity; Child health services; Narration; Child development; Chile Crece Contigo.
Con el retorno de la democracia en Chile, la superación de la pobreza fue asumida como uno de los objetivos más importantes del Estado, en un contexto en que el país dejaba atrás una dictadura militar con un $68 \%$ de pobreza e indigencia (Abufhele, 2019). Este objetivo se implementó vía dos líneas de acción: la estimulación del desarrollo de la economía o el llamado “crecimiento económico" y un nuevo impulso a la política social bajo el sintagma "crecer con equidad". Lo importante de esto es que, si bien los partidos de oposición durante la década de 1980 hicieron una crítica importante a las políticas económicas neoliberales de la dictadura militar, tanto desde el punto de vista económico como de sus costos sociales, la superación de la pobreza no implicó necesariamente una transformación sustancial del modelo, manteniendo la matriz económica, así como una política social, altamente estratificada, basada en estrategias de focalización y asistencialismo estatal para los sectores más pobres.

A partir del primer gobierno de Michel Bachelet, las políticas de salud infantil y otras políticas sociales pasaron a ser uno de los ejes más relevantes de las iniciativas públicas que se debió, en parte, a diagnósticos diversos que daban cuenta de la existencia de mayores índices de pobreza multidimensional en la población infantil y la voluntad política de un sector de la clase política de avanzar hacia un estado de protección social de corte universal y que contrarrestara los efectos de exclusión y reproducción de la pobreza de las políticas de focalización imperante en Chile. Durante su mandato, se estableció como objetivo programático de la salud pública para el período 2011-2010 la superación de los llamados "rezagos y retrasos en el desarrollo psicosocial infantil" con la creación en el 2006 del Sistema de protección de la infancia Chile Crece Contigo (Chile, [2020]) -CCC, de aquí en adelante-. Este sistema, más que crear nuevas ofertas de servicios, articuló los programas dirigidos a la primera infancia desde un giro de una atención sectorial y profundamente desarticulada (Caro, 2009) hacia una atención de tipo integral. Para ello, se fortalecieron las prestaciones entregadas desde el periodo de gestación hasta los 4 años por medio de 
tres líneas de acción: Programa nacional de apoyo al recién nacido, Programa de apoyo al desarrollo biopsicosocial y Programa Educativo para niños/ as en edad preescolar y escolar.

Los escasos estudios realizados en Chile sobre este sistema (Abarca, 2018; Caro, 2009; Castillo, 2016; Vera Rojas; Barra Urquieta; Montes Maldonado, 2018) y nuestros propios avances de investigación sugieren que, pese a que el sujeto de atención es el/la niño/a, gran parte de las prestaciones y actuaciones que el sistema implementa se apoyan fuertemente en el sistema de cuidados próximos al entorno maternal, por medio de la promoción de la lactancia materna, la estimulación temprana y la crianza “activa”, bajo la égida de lo que ha sido en llamar intervenciones en apego y competencias parentales. No se aprecian medidas de corte comunitarias ni más amplia en que los/las niños/as se desenvuelven ni una perspectiva de género que reconozca las desigualdades de género al interior de las propias familias y los obstáculos a nivel estructural con las cuales se enfrentan las mujeres para alcanzar los ideales de salud y bienestar promovidos por esa política. Así el sistema sigue considerando a las madres como el punto de partida en la producción del bienestar infantil (Álvarez, 2013; Anzorena, 2015; Burman, 1998; Castillo, 2016; Contreras Tinoco; Castañeda-Rentería, 2016; Magaña et al., 2011) en un contexto en que las condiciones estructurales de reproducción de la desigualdad han sido escasamente abordadas por el Estado, actuando más bien como una zona de amortiguación de la desigualdad social.

Este sistema también revela las ambigüedades que representan para los objetivos de la salud pública las luchas de las mujeres por la ciudadanía. De este modo, las demandas de los movimientos de mujeres y feministas en torno a la producción de salud y bienestar (Gil-Estevan; Solano-Ruíz, 2017) muestra la tensión entre la pluralidad de modelos de maternidad, identidades de género y la tendencia de los sistemas de salud a reproducir sus formas hegemónicas (Zicavo, 2013).

Teniendo en cuenta la importancia que adquieren las prácticas maternas de cuidado y crianza en ese sistema de atención en salud infantil, presentamos los resultados de una investigación más amplia, en la que se exploran las narrativas de mujeres chilenas usuarias del sistema CCC. El abordaje narrativo nos permitió cartografiar, desde la enunciación del propio yo, las formas en que las subjetividades femeninas se nombran, posicionan, modelan y resisten al dispositivo estatal. Las preguntas que guiaron el análisis fueron: ¿cuáles son las narrativas sobre la maternidad y el cuidado que emergen en los discursos de las usuarias de un dispositivo de atención de la infancia temprana en Chile? y ¿de qué manera estas narrativas reproducen, conflictúan o tensionan al propio sistema?

\section{Método}

El presente estudio formó parte de una investigación mayor que analizó multimodalmente el sistema CCC por medio de una aproximación cualitativa de alcance exploratorio y descriptivo. Los resultados que se presentan corresponden a la fase en que se usó el enfoque narrativo (Bernasconi, 2011; Riessman, 2008; Riessman; Quinney, 2005), que entiende las historias personales como unidades narrativas constructoras de sentido e identidad, entrelazadas con prácticas sociales y relaciones de poder (Salvo Agoglia, 2016).

Las narrativas se constituyen como nuevos objetos de análisis discursivo, atribuyéndoles una importancia relevante en la investigación social y psicológica dada la capacidad de descripción de realidades subjetivas (Biglia; Bonet Martí, 2009). De acuerdo a Reismann (2008), no existe una definición homogénea de narrativa y esa variación está vinculada a los objetos y dimensiones de análisis de las propias disciplinas. Lo que distingue el enfoque narrativo es su aproximación al lenguaje, asumiendo una perspectiva dialógica que pone énfasis en la dimensión heteroglósica y responsiva de cualquier producción lingüística (Balash; Montenegro, 2003). Así, el lenguaje es entendido como una actividad situada contextualmente y que a la vez reproduce una red de relaciones en la cual estamos involucrados. Para Riessman (2008, citado por Salvo Agoglia, 2016, p. 5), se trata "de un proceso permanente de resignificación, lo que habilita conocer las relaciones entre la capacidad de agencia y los discursos y 
estructuras sociales dominantes, recuperando historias invisibilizadas y periféricas". En el contexto de la política social, las narrativas son relevantes, pues permite captar los modos en que los sujetos incorporan representaciones y discursos institucionales presentes en las orientaciones de las políticas públicas, lo cual configura parte de sus discursos a la hora de relatar sus experiencias ante crisis particulares (Arteaga Aguirre; Iñigo Valderrama, 2015).

\section{Participantes}

El grupo de participantes se conformó por medio de una muestra no probabilística opinática (Ruíz Olabuénaga, 2003), constituido por 12 mujeres usuarias del sistema de protección CCC (Tabla 1). Los criterios de selección fueron: mayor de 18 años, al menos un año de antigüedad en el servicio y usuaria de los dos programas principales del servicio (PDBS y PARN). La edad promedio de las entrevistadas es de 30 años, y se presenta un caso de una mujer de 19 años y otra de 50 años, todas residentes de la región metropolitana de Santiago de Chile. Estas fueron contactadas por informantes clave, que siguieron una lógica de muestreo secuencial conceptualmente conducido (Flick, 2004), buscando, a medida que se avanzaba en la ejecución de las entrevistas, el análisis y la comparación constante de los casos, abarcar un conjunto diverso de usuarias de CCC, considerándose progresivamente las condiciones de nivel de estudios, tipo de ocupación y división de tiempos entre trabajo remunerado y labores domésticas.

\section{Tabla I - Participantes}

\begin{tabular}{|c|c|c|c|c|}
\hline & Edad & Ocupación & N. de hijos & Estado civil \\
\hline 1 & 50 & Dueña de casa & 2 & Casada \\
\hline 2 & 32 & Dueña de casa & 2 & Casada \\
\hline 3 & 27 & Dueña de casa & 3 & Conviviente \\
\hline 4 & 24 & Profesional & 1 & Soltera \\
\hline 5 & 30 & Profesional & 1 & Soltera \\
\hline 6 & 36 & Profesional & 2 & Separada \\
\hline 7 & 29 & Profesional & I & Soltera \\
\hline 8 & 30 & Dueña de casa & I & Conviviente \\
\hline 9 & 25 & Estudiante & 2 & Casada \\
\hline 10 & 19 & Desocupada & 1 & Soltera \\
\hline 11 & 22 & Estudiante & 1 & Casada \\
\hline 12 & 47 & Dueña de casa & 6 & Casada \\
\hline
\end{tabular}

\section{Instrumento de producción de información}

El instrumento de producción de información consistió en una entrevista individual semiestructurada de orientación narrativa (Flick, 2004), cuyo objetivo fue que las participantes relataran de modo abierto su experiencia de maternidad relacionada con su vinculación a los programas de CCC, usando una pauta-guía flexible de preguntas con los temas y subtemas por explorar a partir de los objetivos de la investigación. Se puso especial atención a las experiencias de las participantes en relación a las atenciones de los profesionales en terreno, su asistencia a talleres, charlas informativas o sesiones con sus hijo/as en sala de estimulación, dependiendo del caso. El guion incluía, además de los tópicos referidos, elementos biográficos (estudios, trabajo, vida de pareja) y preguntas reflexivas en torno a la maternidad y la crianza en formas de historias, recuerdos, ejemplos, etc. 
Las entrevistas fueron realizadas el año 2018 por la investigadora y su equipo en los domicilios o lugares públicos cercanos a las entrevistadas en Santiago de Chile, y tuvieron cada una en promedio una hora de duración. Además de la entrevista, se completó una ficha sociodemográfica, donde se consignaron la edad, ocupación, nivel educacional, número de hijos, estado civil y personas con las que conviven la madre y sus hijos/as. Todas las entrevistas fueron grabadas en audio y transcritas en su totalidad, tuvieron previa aprobación y firma por parte de las entrevistadas y los entrevistadores de un consentimiento informado visado por el comité de ética de la institución patrocinante de la investigación (Informe Comité de Ética 29/2019). En este se señalaban los objetivos del estudio, los procedimientos, garantizando la confidencialidad y el anonimato en la publicación de la información.

\section{Análisis de datos}

Como señala Reissman (2005), no existe un procedimiento homogéneo para el análisis de las narrativas. Esta libertad posibilitó triangular dos aproximaciones analíticas ya probadas en otro estudio (Calquin; Cerda; Yáñez-Urbina, 2018) y que permitió, desde nuestra perspectiva, una mayor profundización de las narrativas en su dimensión semántica como pragmática: el análisis temático (Reissman, 2005) y una estrategia general discursiva (Potter; Wetherell, 1987). Ello hizo que la interpretación de la narrativa se dirigiera tanto a identificar núcleos temáticos narrativos como a diferenciar algunos elementos pragmáticos del lenguaje, considerando a las explicaciones y tipos de narrativas como formas de acción social. Dado el carácter exploratorio y el tamaño de la muestra, se llevó a cabo una aproximación general de las narrativas, sin considerar comparaciones por estrato económico, edad o conformación familiar, pese a que los resultados permitieron visualizar ciertas variaciones y convergencias en relación a estas variables de estratificación social.

El análisis tuvo dos etapas: un análisis intracaso, dirigido a reconstruir los elementos narrativos propios de cada entrevista; y un análisis intercasos o transversal efectuado para develar lógicas narrativas comunes en base a categorías emergentes, como las formas de narración, experiencias comunes en atenciones y talleres, relaciones con los profesionales, servicios y productos ofrecidos por CCC y, finalmente, significados sobre maternidad y crianza. Así, las narrativas fueron reconstruidas con base en cuatro elementos transversales presentes en las entrevistas, tres de contenido: imagen del yo materno, relación del sujeto con el Estado, relaciones de la usuaria con los productos y servicios prodigados por CCC y uno de tipo formal-pragmático: la conformación temporal de la exposición narrativa (Gergen, 1997).

Una vez identificadas y reconstruidas las narrativas, se procedió a hacer la síntesis de los resultados y triangular la información con el marco teórico.

\section{Resultados}

Se presentan tres narrativas identificadas en los relatos de las mujeres entrevistadas y que se ejemplifican en extractos que resultaron ser los más representativos y sintéticos de los elementos que estructuran cada narrativa, esto en función de destacar la trama estructurante de los relatos -más que los contenidos- propio de este enfoque y que lo distingue de otras aproximaciones cualitativas (Balash; Montenegro, 2003; Bernasconi Ramírez, 2011; Riessman, 2008). Cabe destacar que en la presentación de los extractos se tradujeron algunos modismos locales para asegurar una mayor comprensión del texto y se identificó a las entrevistadas por otros nombres para preservarles su anonimato.

\section{Yo materno converso}

Esta modalidad narrativa revela la importancia que tiene para la experiencia de la maternidad los conocimientos transmitidos por el sistema CCC, así como los objetos que este prodiga a las familias usuarias (cunas, ajuares, libros, folletos, etc.) Así la historia del embarazo, el parto y la crianza es relatada por las mujeres por medio de una estructura temporal que Gergen (1997) llama de tipo progresiva y que se plantea desde un tránsito de un estado de ignorancia o no-saber a un estado 
de saber e inclusive de felicidad. Esta progresión hacia el saber (de los cambios y evolución del embarazo, de los riesgos y alternativas de parto y desarrollo infantil, entre otros aprendizajes) va adquiriendo un particular significado en la vida emocional de las mujeres al ir entretejiéndose con el problema del deseo materno, que se pone en juego por medio de esta misma estructura de progreso y transformación: el paso, para algunas entrevistadas, de un embarazo no deseado al deseo de continuar el proceso y convertirse en madre, tal como se aprecia en este relato.

Y sí, si igual era como superimportante, uno que es como primeriza y si bien una igual tiene un poco, algo de noción del tema del embarazo y todo, era distinto cuando una está embarazada y va viendo el libro, con las etapas de que va a pasar después, como que ahí te entusiasma un poquito. (Noelia)

Para situar contextualmente esta narrativa, es necesario precisar que el sistema identifica como factor de riesgo lo que denomina "conflicto con la maternidad" evaluado por dos preguntas: intentos de aborto y toma de decisión del embarazo. La intervención, en su etapa temprana, considera asesoría e intervención psicológica a aquellas madres que han intentado abortar (en un contexto nacional en que el aborto no terapéutico es ilegal) o no han tomado la decisión de quedar embarazada.

En esta narrativa, información y deseo son significantes mutuamente dependientes: el deseo materno se organiza como experiencia móvil que puede ser resignificada por medio de intervenciones informativas y terapéuticas. De este modo, estos tránsitos articulados del no-deseo al deseo y del no-saber al saber son sustanciales para la implementación y éxito inicial del propio sistema y para el posicionamiento de las mujeres como sujetos beneficiarios del CCC, pues de ocurrir lo contrario, es decir, el rechazo al embarazo o al recién nacido, se activan los circuitos de derivación hacia otros sistemas de protección -por ejemplo, adopción-.

Para esta narrativa, un aspecto sustancial son los objetos que el sistema ofrece a las mujeres, los cuales revelan tomar un lugar particular y privilegiado en el proceso de conversión y funcionan como anzuelos para la adherencia y reconocimiento del sistema en la población general y, además, como una ortopedia para la crianza. Por ejemplo, acá se revelan como elementos para un estado de felicidad:

Ya mira, en el primer ajuar, igual era bonito, era bien completo porque era la primera vez que te daban cuna, te dabas cuenta, ya que con te den la cuna las mamás eran felices, te daba lo mismo ya lo que trajera el ajuar porque ya una cuna es otra cosa. (Karina)

Estos objetos se inscriben como elemento de los proyectos de gestión del propio yo y regulación del deseo materno y un juicio hacia el sistema como prestador de servicios tangibles, novedosos e incluso con ciertas propiedades estéticas.

Véase esto en otro fragmento de una entrevista:

[CCC] está llegando a grupos de gente que está como interesada... ahora, no sé, son como harta gente de menos recursos. No sé, ya si de menos recursos, yo igual me incluyo en los menos recursos ya. Ahora cuál es la gracia de esto, que el Chile crece contigo te entrega de la cuna para arriba. Entonces no hay excusa, aunque el bebé hay nacido en un espacio que era... desprovisto de posibilidades va igualando desde la base, desde lo primordial... como las capacidades del niño. A que voy... por ejemplo, ya la guagüa [bebé] recibe una cuna, entonces en su casa, aunque no haya camas, va a tener una cuna, va a tener un espacio para él. Tiene un móvil, porque la primera forma de estimulación para el bebé es un móvil... entonces vamos como igualando el asunto. Nadie va a decir que un cabro [joven] que nace en la pobla [barrio popular] va a tener las mismas capacidades que otro, pero por lo menos esto permite que eso disminuya en el cerebro de una guagua, o sea cuando está maleable ¿cachai? [isabes?], lo importante [es] que tenga estimulación y que tenga así, como lo mínimo, abrigo, calor, cariño y ese tipo de cosas. (Mónica)

En este extracto, vemos que la entrevistada se integra lingüísticamente a una colectividad de mujeres/madres que denomina "con menos 
recursos" y que son, o pueden ser, beneficiarias del programa estatal. El sujeto hablante logra así incorporar en su narración otros sujetos imaginarios e hipotéticos, desconocidos, abstractos, que comparten una misma comunidad de mujeres. En este sentido, el acceso a los productos promueve una percepción más o menos generalizada en las mujeres entrevistadas con mayores condiciones de igualdad, o como se dice en el texto de "igualación del asunto", entre sectores más pobres y sectores más privilegiados de la población y en que las condiciones estructurales de desigualdad tienen un rol ancilar.

\section{Yo materno emprendedor}

Esta narrativa, si bien presenta una forma de tipo ascendente como la anterior, se destaca por la identificación de una subjetividad materna modelada por los discursos e imaginarios expertos (Illouz, 2009). Está marcada por lo que Imaz (2010) llama maternidad bricoleur, en la cual se articula el discurso tradicional de la maternidad como instinto natural al discurso de la elección, el discurso experto y el discurso del empoderamiento (Salvo Agoglia, 2016). A diferencia de la narrativa anterior, en esta la maternidad se posiciona a partir del deseo y el saber, y es concebida como un emprendimiento personal que anima a buscar espacios de autoformación alternativas al modelo biomédico. Al respecto, véase el siguiente fragmento:

Yo desde siempre, por interés personal, así como de muy chica me gustaron siempre el tema madre, hijo, guagüa, ese era como mi tema [...]. Claro, ya veníamos con el primer hijo nacido, de esta manera [se refiere al apoyo de Dulas] conversamos con alguien, para todos era como “iWoh!", pero también después por ejemplo yo hice el curso de Dula cuando empecé a formalizar en esta... yo ese curso lo hice embarazada del segundo hijo, entonces yo sí o sí estaba pensando parto en casa y para todo el mundo era como terrible como: "ino!, iqué irresponsable!", casi que "estai loca”... Entonces dijimos "ilo vamos a hacer dentro que se pueda!, si hay alguna complicación nos vamos al hospital o a la clínica y listo". (Alejandra)
Esta actitud de empoderamiento expresado en el relato también expone la actitud subjetiva hacia los objetos y productos de estimulación entregados por el sistema. Recordemos que en la narrativa estos productos son fundamentales; en este caso, los objetos son evaluados desde una posición clientelar y distante. La misma entrevistada, al ser consultada sobre estos productos, señala lo siguiente:

Yo creo que sí, pero de nuevo, como decía, el usuario tiene que ser activo... porque te pueden entregar el montón de cuestiones y tú las guardai en el closet... o... hay gente que las vende [risas] en la feria o por Facebook, ¿te das cuenta? [...] Pero no, yo creo que con uno principalmente con uno ocuparlo el sacarle provecho y si a veces creo que hay que ponerle un poquitito más de... (Alejandra)

Si en la narrativa anterior el objeto por sí mismo genera un buen desarrollo infantil, en esta la acción de las madres es fundamental; se trata de la importancia que adquiere el comportamiento de las usuarias para el éxito de la intervención. Lo interesante de esta narrativa es que la acción emprendedora queda determinada por representaciones en torno a la clase social. En la narrativa anterior hay un intento de identificación con el resto de las mujeres usuarias en lo que llamamos la comunidad de mujeres -somos madres de bajos recursos- en esta narrativa el uso del verbo activo se conforma como un punto de inflexión y cisura de esa comunidad. Esta vez es una voluntad de diferenciación respecto a las clases populares, así la misma entrevistada continúa señalando que: $P a$ 'sacarle bien el provecho con un poquito más de capital, no sé, como cultural la familia yo creo... pero eso igual le pueden sacar provecho en familias donde a lo mejor no hay muchos estudios que sé yo, igual se puede (Alejandra).

Esta producción de diferencias connata dos cuestiones. Por un lado, una representación del sujeto pobre como pasivo, con un déficit del actuar emprendedor -crianza y uso de objetos de forma activa- y que el Estado debe fomentar; por otro, una representación de la maternidad en contextos de pobreza que se basa en la creencia extendida y dominante de una cultura parental de la pobreza (Main; Bradshaw, 2016) según la cual, las personas en 
situación de pobreza desplegarían prácticas de crianza y cuidados muy distintas a las personas de otros grupos sociales, diferencia que a su vez se valora como negativa y es asociada a un modelo de déficit parental.

\section{Yo materno ambivalente}

En esta narrativa se describe un posicionamiento ambivalente hacia el sistema CCC. Hay tanto un reconocimiento de las virtudes y utilidad de los servicios o adminículos brindados, pero también se destacan aspectos críticos en varias dimensiones y niveles de criticidad.

Entonces yo creo que está bueno. Lo único que encuentro es que ya cuando nace la guagua son como muy estructura'os así como, a estos meses tiene que hacer esto, a estos meses la guagua tiene que hacer esto. (Sofía)

O sea yo creo que es como lo mismo, que es como un ente vigilante ¿cachai?, como en ese sentido, como o no sé, o sea así como a ver... es que cuática [complicado] igual, así como medio pasivo-agresivo, así como "mamita, usted tiene que hacer esto" ¿cachai? Ponte tú, yo igual me acuerdo que cuando yo quedé embarazada y conté que había tratado de abortar y me mandaron a la psicóloga, después fue una trabajadora social a mi casa, de la nada, de repente sonó el timbre, "soy la trabajadora social del consultorio", y ahí tú dices "ichucha!”[expresión para connotar sorpresa]. (Andrea)

Apreciamos tanto una crítica a los esquemas rígidos del desarrollo infantil que el sistema transmite como su función de vigilancia y control del deseo y las prácticas maternas. De esta forma, se erigen gestos de resistencia, algunos más conscientes que otros, que logra fisurar los sentidos hegemónicos circulantes del CCC de la maternidad en tanto, de acuerdo a Rich (2019), institución normativa. Esta narrativa expresa la experiencia compleja de lo materno, que desarrolla esta misma autora: diversa y personal. Se desarrollan dos repertorios discursivos (Potter; Wetherell, 1987), incluso opuestos y que en el extracto siguiente adquiere la forma de una descripción normativa de la lactancia a la vez que, al final del extracto, se desliza una fuga de sentido; esto por medio de un reparo sustancial que incorpora el verbo rechazar.

Yo sentía que no le daba solo leche, sino que le daba amor con eso ¿cachai? [...] porque también es distinto cuando que a mí también me pasó un tiempo, cuando te genera un rechazo que el niño esté tomando pecho, tú dices por qué, incómodo que una guagua esté en una pechuga. (Andrea)

\section{Discusiones y conclusiones}

El presente estudio se planteó como objetivo identificar y analizar las narrativas sobre la experiencia de la maternidad en el contexto de intervención de un sistema de atención de la infancia temprana en Chile y se propuso responder a las preguntas acerca del tipo de narrativa que emerge, así como los posicionamientos de las mujeres respecto al sistema. El recorrido analítico realizado muestra que, si bien hay una tendencia a designar a las mujeres/madres como beneficiarias naturales de las políticas sociosanitarias en la infancia temprana, estas mujeres cuentan con grados de autonomía respecto a asumir los modelos y demandas que el sistema de salud promueve. Las narrativas reconstruidas nos permitieron conocer múltiples posicionamientos, agenciamientos, comprensiones y horizontes desde los cuales las mujeres se sitúan en el dispositivo sanitario en cuestión.

El análisis de las entrevistas dio como resultado la presencia de tres narrativas que organizan las palabras de las mujeres y que en algunos casos se hace presente en una misma entrevista. Es necesario destacar que las narrativas no corresponden a un sujeto en particular (Balasch; Montenegro, 2003) del cual emanan las enunciaciones, ni meras reproducciones del orden social, sino que tratan de voces que habitan a los sujetos y que responden a multiformes enunciaciones del yo que se construyen en el mismo encuentro entre investigadores y participantes. En este contexto, el análisis nos permitió indagar en un yo que se revela no solo como construcción, relación y narración, sino que además como multiplicidad. 
Las tres narrativas que denominamos conversión, emprendedora y ambivalente expresan formas y operaciones de producción discursiva complejas y en el que destacan diversos repertorios y que a partir de la pregunta por la maternidad nos lleva a cuestiones como la función del Estado, la pobreza, la crianza, la relación mujer-Estado-sociedad y de las mujeres consigo mismas. Un resultado interesante fue el lugar privilegiado que adquieren los objetos prodigados por el sistema para organizar las narrativas.

La narrativa de la conversión permite, según Foucault (2006), circunscribir el arte de gobernar a formas de señalamiento del poder vinculado a la experiencia de revelación -saber y deseo- y acompañamiento del devenir materno. Se trata, por un lado, de un proceso de guía de conciencia que se aprecia en cuestiones como derivación hacia profesionales de la salud mental de las mujeres que declaran "conflicto con la maternidad" y cuyo objetivo es la continuación del embarazo; también, en la importancia de los conocimientos biomédicos para alcanzar un ideal normativo de la maternidad contemporánea asociada al manejo de información científica y experta (Ehrenreich; English, 2010), y finalmente la importancia de los objetos para alcanzar ciertos estados de felicidad materna y adherencia a las prestaciones.

Es una la narrativa en que se destaca el acompañamiento experto o formas más blandas de control. Como dice Bröckling (2015, p. 54), "para llegar a saber qué se es, se requiere aparentemente a alguien que se lo diga". Lo interesante en esto es que el sistema despliega la paradoja de la subjetivación ayudada por expertos: por un lado, esta forma narrativa valida el propio saber, a la vez asume como verdad los saberes expertos, que pensamos se vinculan a formas de sostener la incertidumbre de la crianza.

Por otro lado, las narrativas emprendedoras forman parte de un discurso cultural y económico desplegado en los últimos años, basado en un modelo empresarial de conducir la subjetividad. Se trata de operaciones que atienden al cultivo de un self emprendedor (Bröckling, 2015) o un empresario de sí mismo (Foucault, 2006), en que el sujeto debe ser autónomo y activo y "cuya confianza por su propia capacidad debe ser reforzada y en forma permanente" (Bröckling, 2015, p. 28). Cabe destacar que esta narrativa es la que plantea mayores críticas al sistema de atención, mostrando y denunciando inclusive las modalidades más duras del poder médico-ginecológico -incluso algunas hablan de vigilancia y castigo-. Concluimos que este yo emprendedor que emerge en los relatos es una resistencia a los sistemas de gestión estatal que lo promueven, si estos se basan en modelos disciplinarios y rígidos que intentan capturar las conductas y conducirlas hacia un solo proyecto de maternidad.

Las narrativas ambivalentes destacan por su complejidad y revelan que las mujeres son capaces de inventar y reinventar sus propias maternidades por medio de confrontaciones constantes a los automodelamientos impuestos, lo que lleva a una formación heterogénea de los modelos y experiencias de las maternidades en las mismas sujetas, mostrando la subjetivación como una multitud de autoinvenciones. Así apreciamos que esas narrativas se aproximan a otros hallazgos (Eriksson et al., 2016; Farfán-Santos, 2019) que señalan que las mujeres otorgan constantemente nuevos sentidos a sus prácticas cotidianas de la maternidad en un contexto cultural de precariedad y transformación aceleradas y constantes, con efectos en la propia capacidad de reconocerse como madres haciendo uso de un entramado de discursos que circulan en la cultura actual y mostrando también el fracaso de los discursos totalizantes que el sistema intenta instalar.

Finalmente, es necesario destacar que el estudio plantea limitaciones en términos de la generalización de sus resultados, especialmente en contextos no urbanos, donde suponemos que ciertos discursos aún no son dominantes en la vida cotidiana de las mujeres como en el contexto urbano-metropolitano y porque el mismo sistema presenta diferencias en su implementación. También, profundizar en el rol que juega la clase social, la edad y la orientación sexual en la producción narrativa, como una forma de multiplicar las diferencias en el ejercicio de la maternidad.

\section{Referencias}

ABARCA, G. “¡Promueva el apego!”: sobre la maternidad de mujeres haitianas como objeto de gobierno en Chile. Bricolaje, Santiago, n. 3 , p. 12-21, 2018. 
ABUFHELE, V. La política de la pobreza y el gobierno de los asentamientos informales en Chile. Eure, Santiago, v. 45, n. 135, p. 49-69, 2019.

ÁLVAREZ, B. La maternidad: entre la decisión individual y/o la obligatoriedad social. In: LÓPEZ MATHEU, C.; BESTARD, J.; MARRE, D. (Ed.). Maternidades, procreación y crianza en transformación. Barcelona: Marcial Pons, 2013. p. 219-242.

ANZORENA, C. ¿Qué implica la protección social para las mujeres? Un análisis feminista de las políticas sociales y de igualdad en Argentina. Oxímora: Revista Internacional de Ética y Política, Barcelona, n. 7, p. 98-118, 2015.

ARTEAGA AGUIRRE, C.; IÑIGO VALDERRAMA, I. Políticas sociales, modelo de desarrollo y subjetividad de grupos vulnerables en Chile. Revista del CLAD Reforma y Democracia, Caracas, n. 61, p. 209-234, 2015.

BALASCH, M.; MONTENEGRO, M. Una propuesta metodológica desde la epistemología de los conocimientos situados: las producciones narrativas. Encuentros en Psicología Social, Málaga, v. 1, n. 3, p. 44-48, 2003.

BERNASCONI RAMÍREZ, O. Aproximación narrativa al estudio de fenómenos sociales: principales líneas de desarrollo. Acta Sociológica, Ciudad de México, n. 56, p. 9-36, 2011.

BIGLIA, B.; BONET MARTÍ, J. La construcción de narrativas como método de investigación psicosocial. Prácticas para una escritura compartida. Forum: Qualitative Social Research, Berlín, v. 10, n. 1, p. 1-25, 2009.

BRÖCKLING, U. El self emprendedor: sociología de una forma de subjetivación. Santiago: UAH, 2015.

BURMAN, E. La deconstrucción de la psicología evolutiva. Madrid: Visor, 1998.

CARO, P. Análisis del programa Chile Crece Contigo desde los ideales normativos y la noción de prueba. Revista de Trabajo Social, Santiago, n. 77, p. 25-37, 2009.

CALQUIN, C.; CERDA, J.; YÁÑEZ-URBINA, C. La producción del feto como "sujeto de derechos": análisis cualitativo de los discursos médicocatólicos en Chile. Salud Colectiva, Buenos Aires, n. 3, p. 391-403, 2018.

CASTILLO, P. Los saberes psicológicos en el neoliberalismo: el caso de las políticas sociales y la teoría del apego en Chile. Universitas Psychologica, Bogotá, v. 14, n. 4, p. 1325-1337, 2016.

CHILE. Acerca de Chile crece contigo. Chile crece contigo, Santiago, [2020]. Disponível em: <https:// bit.ly/30xi7Ok>. Acesso em: 14 out. 2020.

CONTRERAS TINOCO, K. A.; CASTAÑEDARENTERÍA, L. I. Tensiones entre el cuerpo productivo de la mujer y la normatividad de género en torno a la maternidad. Revista Latinoamericana de Estudios sobre Cuerpos, Emociones y Sociedad, Buenos Aires, v. 8, n. 21, p. 10-24, 2016.

EHRENREICH, B.; ENGLISH, D. Por tú propio bien: 150 años de consejos expertos a las mujeres. Barcelona: Capitán Swing, 2010.

ERIKSSON, E. et al. When they talk about motherhood: a qualitative study of three groups' perceptions in a Swedish child health service context. International Journal for Equity in Health, New York, v. 15, n. 99, p. 1-10, 2016.

FARFÁN-SANTOS, E. La política de la resistencia: el acceso a los servicios de salud y la maternidad de las mexicanas indocumentadas en los Estados Unidos. Latino Studies, Londres, v. 17, n. 1, p. $67-85,2019$.

FLICK, U. Introducción a los métodos cualitativos de investigación. Madrid: Morata, 2004.

FOUCAULT, M. La voluntad de saber. Ciudad de México: FCE, 2006.

GERGEN, K. Realidad y relaciones. Barcelona: Paidós, 1997.

GIL-ESTEVAN, M.; SOLANO-RUÍZ, M. C. Diversidad cultural y lactancia materna: prestación de cuidados culturalmente competentes en atención primaria. Index de Enfermería, Granada, v. 26, n. 3, p. 162-165, 2017. 
ILLOUZ, E. La salvación del alma moderna:

terapia, emociones y la cultura de la autoayuda.

Madrid: Katz, 2009.

IMAZ, E. Convertirse en madre: etnografía del tiempo de gestación. Madrid: Cátedra, 2010.

MAGAÑA, I. et al. Diversidad familiar, relaciones de género y producción de cuidados en salud en el modelo de salud familiar: análisis de caso en un Cesfam de la región metropolitana, Chile. Terapia Psicológica, Santiago, v. 29, n. 1, p. 33-42, 2011.

MAIN, G.; BRADSHAW, J. Child poverty in the UK: measures, prevalence and intra-household sharing. Critical Social Policy, London, v. 36, n. 1, p. 1-24, 2016.

POTTER, J.; WETHERELL, M. Discourse and social psychology: beyond attitudes and behaviour. London: Sage, 1987.

RICH, A. Nacemos de mujer: la maternidad como experiencia e institución. Madrid: Traficante de Sueños, 2019.
RIESSMAN, C. K. Narrative methods for the human sciences. Boston: Sage, 2008.

RIESSMAN, C. K.; QUINNEY, L. Narrative in social work: a critical review. Qualitative Social Work, Londres, v. 4, n. 4, p. 391-412, 2005.

RUÍZ OLABUÉNAGA, J. I. Metodología cualitativa. Bilbao: Deusto, 2003.

SALVO AGOGLIA, I. Construcción de la maternidad en adopciones monoparentales: mandatos, deseos y elecciones. Psicología, Santiago, v. 25, n. 2, p. 1-18, 2016.

VERA ROJAS, W. D.; BARRA URQUIETA, C.; MONTES MALDONADO, C. El enfoque institucional del cuidado en infancia: Chile y Uruguay. Perfiles Latinoamericanos, México, v. 26, n. 52, p. 1-27, 2018.

ZICAVO, E. Dilemas de la maternidad en la actualidad: antiguos y nuevos mandatos en mujeres profesionales de la ciudad de Buenos Aires. La Ventana, Guadalajara, v. 38, n. 4, p. 50-86, 2013.

\section{Contribución de los autores}

Calquin es la directora responsable de la investigación de la cual se obtuvieron los resultados. Contribuyó al diseño e implementación de la investigación, análisis de datos y estructura y escritura del manuscrito. Guerra contribuyó a la implementación de la investigación, coordinación del trabajo de campo, análisis de datos y estructura del manuscrito. Vásquez contribuyó a la implementación de la investigación, recogida de datos y redacción del manuscrito. Todos los autores discutieron los resultados y contribuyeron a la versión final del manuscrito.

Recibido: 13/04/2020

Aprobado: 30/07/2020 